\title{
A Review on Plastic Behavior of Polymer Sheets and Forming Process
}

\author{
Eun Ho Lee* \\ School of Mechanical and Control Engineering, Handong Global University, Republic of Korea
}

Submission: June 7, 2018; Published: July 05, 2018

*Corresponding author: Eun Ho Lee, School of Mechanical and Control Engineering, Handong Global University, Pohang, Republic of Korea, Tel: +82-54-260-1365; Email: ehlee@handong.edu

Abstract

This paper presents a mini review about plastic behaviour of polymer sheets. The fundamental of plasticity and concept of constitutive modeling are introduced. As an application of the plastic behaviour, studies on forming process of polymer sheets are presents. Finally, the future woks are also discussed in the conclusion section.

Keywords: Polymer; Plastic behaviour; Forming; Target temperature; Lightweight; Metal flow

\section{Introduction}

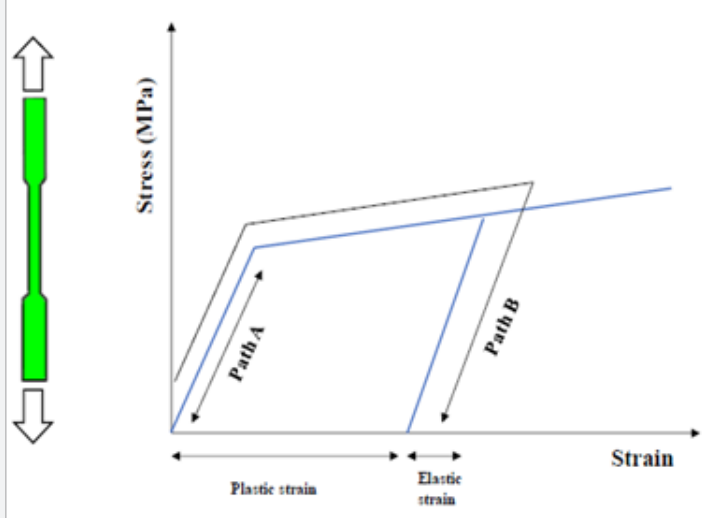

(a) 1-dimensional Tensile test

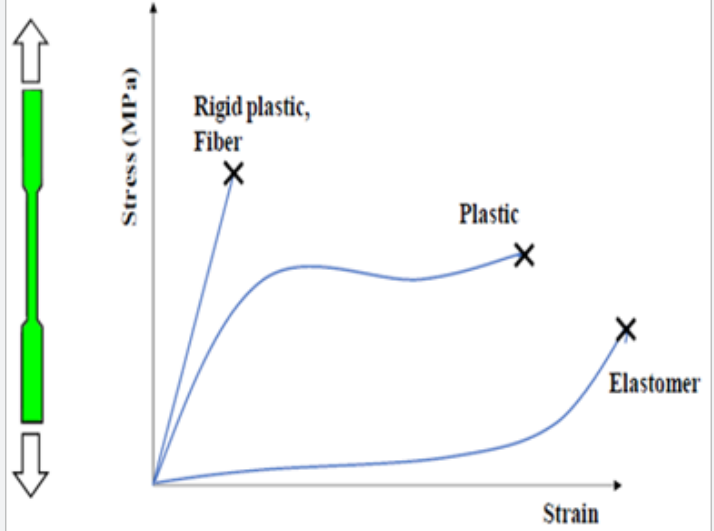

(b) Mechanical behaviors of polymer sheets

Figur 1: Plastic behavior of polymer sheets.

Polymer is one of widely used material due to the great availability and low cost. For mass production, moulding and extrusion technologies have been widely employed for polymer processing in industries [1]. Recently, automotive industries are trying to reduce the weight of the vehicles for improving the fuel efficiency [2]. For the reason, they have an interest in polymer materials, and many researchers have studied mechanical behavior of polymer materials. Figure 1(a) shows an example of 1-dimensional tensile test which is the most widely used material testingmethods to obtain the material's behavior. When a material is getting deformed, the material has aproportional relation between stress and strain within a range of strain and this proportional relation iscalled elastic modulus. This strain rage is called elastic range and the stress is called yield stress. In theelastic range, the deformed material can recover the original shape, as shown in the path A. However, if thestrain level is over the elastic limit, a permanent deformation remains on the material even though thematerial slightly recovers the strain $[3,4]$. This recovered strain is called elastic strain, and the permanentdeformation is called plastic strain, as shown in path 
B of Figure 1(a). Figure 1(b) shows general behaviors ofpolymer materials. The elastic behavior is almost the same for all the materials. However, right after theelastic limit, rigid plastic easily leads to a material failure while other materials have more margin to bedeformed. For the reason, rigid plastic material does not Table 1: Mechanical property of polymer materials [5]. show plastic behavior. This break point determinesthe elongation, the ration between the deformed length and the original length until the break point. Table 1 shows a summary of the mechanical property of polymer materials [5].

\begin{tabular}{|c|c|c|c|}
\hline Polymer Type & Ultimate Tensile Strength (Mpa) & Elongation (\%) & Elastic Modulus (GPa) \\
\hline ABS & 40 & 30 & 2.3 \\
\hline Acetal Copolymer & 60 & 45 & 2.7 \\
\hline Acrylic & 70 & 5 & 3.2 \\
\hline Nylon 6 & 70 & 90 & 1.8 \\
\hline Polyimide & 85 & 7 & 2.5 \\
\hline Polycarbonate & 70 & 100 & 2.6 \\
\hline Polyethylene Terephthalate (PET) & 55 & 125 & 2.7 \\
\hline Polypropylene & 40 & 100 & 1.9 \\
\hline Polystyrene & 40 & 7 & 3 \\
\hline
\end{tabular}

During the plastic deformation, the stress-strain relation is given as below [6,7]:

$$
d \sigma=D d \varepsilon^{e} d \varepsilon \text { where } d \varepsilon^{e}=d \varepsilon-d \varepsilon^{p},
$$

$$
\begin{aligned}
& \bar{\sigma}(\sigma)=\rho\left(\bar{\varepsilon}^{p}\right) \\
& \sigma_{n+1}=\sigma_{n}=d \sigma_{n+1} \\
& \rho_{n+1}\left(\bar{\varepsilon}^{p}\right)=\rho_{n}\left(\bar{\varepsilon}^{p}\right)+H d \bar{\varepsilon}^{p}
\end{aligned}
$$

then the increment of the plastic strain is given with the flow rule,

$$
d \varepsilon^{p}=\frac{\partial q}{\partial \sigma} d \bar{\varepsilon}^{p}
$$

$d \sigma$ is the increment of stress, $D$ is the elastic modulus matrix, $d \varepsilon$ is the increment of total strain. $d \varepsilon^{e}$ represents the increment of elastic strain, $d \varepsilon^{p}$ is the increment of the plastic strain. $q$ is the plastic potential function, and $d \bar{\varepsilon}^{p}$ is the equivalent plastic strain. In order to obtain the $d \varepsilon^{p}$, below three conditions should be employed, $\bar{\sigma}$ is equivalent stress, $\rho$ is flow stress model, and $H$ is the slope of stress-strain curve. Equation (3-1) is the yielding condition, (3-2) is about the return mapping stress, and (3-3) is the hardening behavior. Solving the equations from (3-1) to (33) provides the increment of the plastic strain and stress. Then the strain and stress are used in numerical analysis of the plastic behavior.

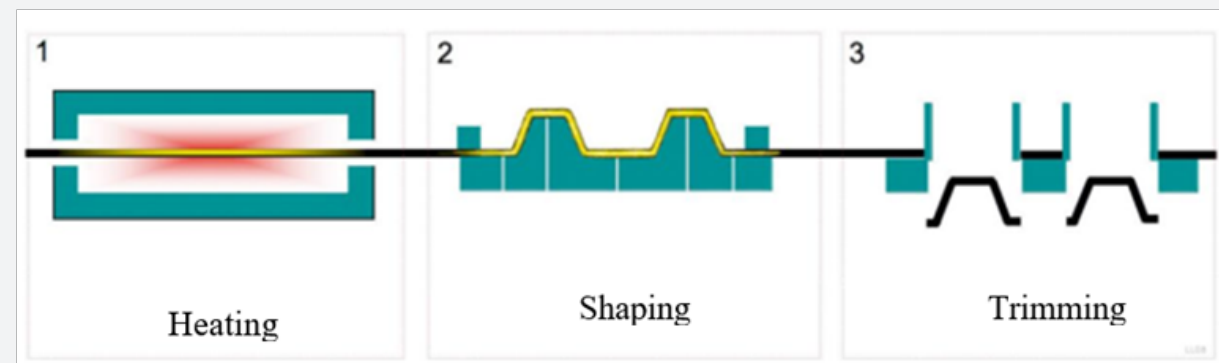

Figur 2: Thermoforming process [8].

As an application of the plastic behavior, some researchers have developed forming technologies for polymer materials. Thermoforming is one of the most popular forming processes for polymer sheets. As shown in Figure 2 [8], the thermoforming process consists of three steps; heating, shaping and trimming. Withthe thermoforming, a polymer sheet is heated to a target temperature, then formed to a specific shape in a mold. The formed sheet is trimmed to make a product. The vacuum forming is very similar to the thermoforming. In the vacuum forming, a polymer sheet is heated to a target temperature, then stretched onto a mold, and forced against the mold with a vacuum condition to reach the target shape.

The thermoforming and vacuum forming requires a mold to form a polymer sheet to the target shape. The investment cost of the mold is expensive. In addition, a mold cannot be used for 
another shape. To reduce the cost, some researchers started studying incremental forming for polymers [9,10]. Figure 3 shows the concept of the incremental forming. A polymer sheet is locally deformed by a forming tool which moves on the surface of the polymer sheet. Since the moving tool is following a defined path in 3-dimensional space, this forming process is flexible to be applied to arbitrary shapes.

Some researchers have tried to build lightweight structures with polymers sheets. Figure 4(b) shows a pyramidal kagome structure made of a polypropylene sheet. Since the polymer sheet was not able to be deformed to reach the target shape in room temperature, a heated die set was employed to increase the formability of the polymer sheet, as shown in Figure 4(a). This study showed a possibility to make a complex shape by a polymer sheet forming process. Polymer sheets can be employed as a medium material to help metal flow in sheet metal forming processes. Figure 5 shows a viscous pressure bulge (VPB) test under biaxial state of stress. Since the viscous medium help the flow of the sheet metal, the VPB forming process was able to reach large strain state. As shown in above examples, forming process of polymer sheets has many chances to be employed in industrial applications.

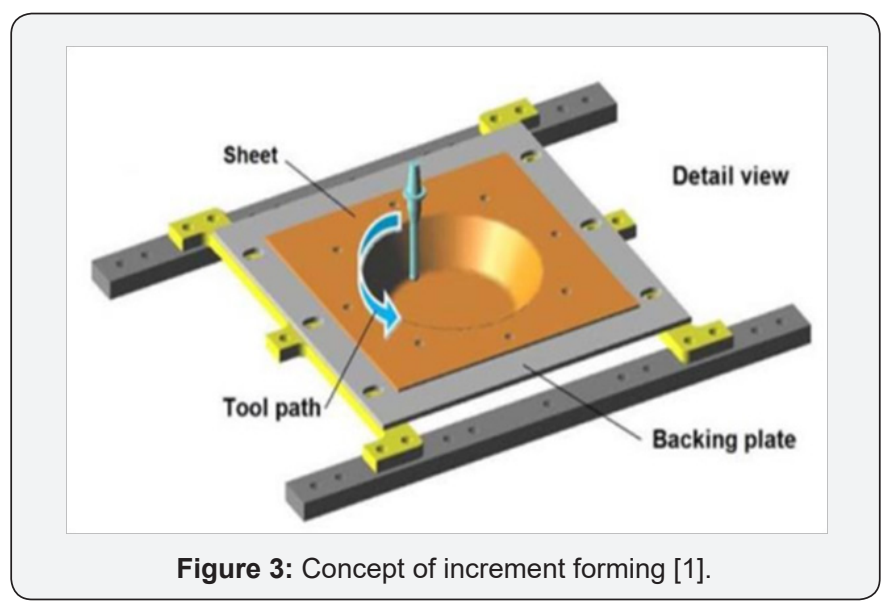

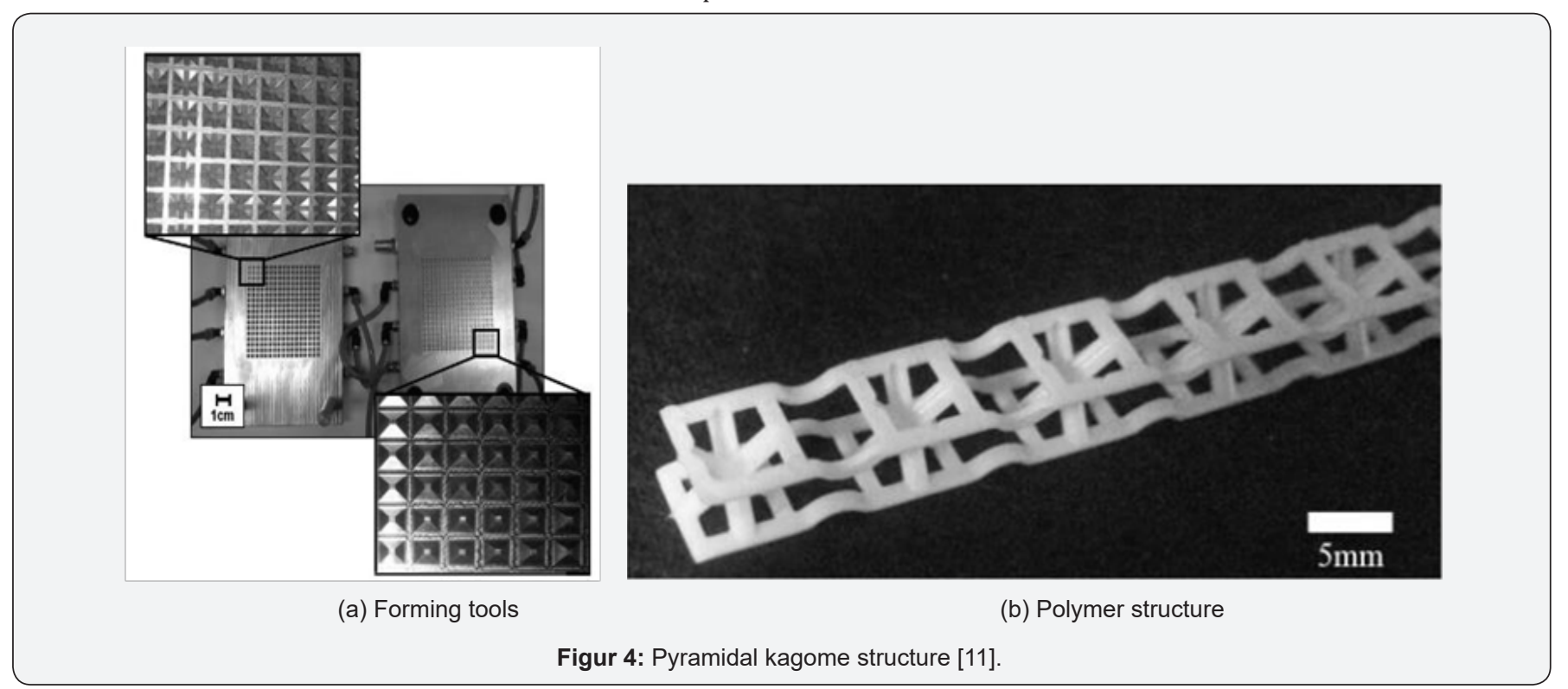

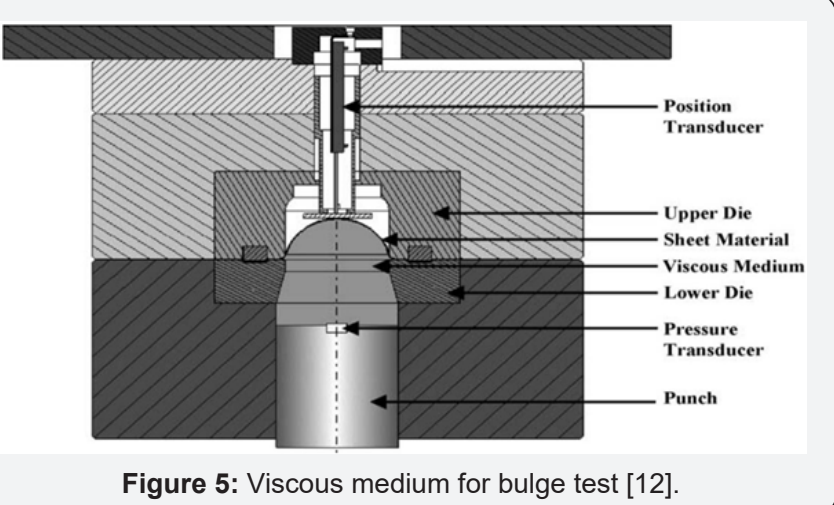

\section{Conclusion}

This paper briefly reviews the plastic behavior of polymer materials and forming technologies as an application. To make more application of the plastic behavior, forming limit [13] and joining technologies [14] should be accounted. Because of the low cost and great availability, the demand of polymer is getting growing, and both fundamental and practical studies are required [15].

\section{References}

1. Martins PAF, Kwiatkowski L, Franzen V, Tekkaya AE, Kleiner M (2009) Single point incremental forming of polymers. CIRP Annals Manufacturing Technology 58(1): 229-232.

2. Lee EH, Hwang JS, Lee CW, Yang DY, Yang WH (2014) A local heating method by near-infrared rays for forming of non-quenchable advanced high-strength steels. J Mater Process Tech 214(4): 784-793.

3. Lee EH, Yang DY, Yoon JW, Yang WH (2015) Numerical modeling and analysis for forming process of dual-phase 980 steel exposed to infrared local heating. Int. J. Solids Struct 75-76: 211-224.

4. Lee EH, Yoon JW, Yang DY (2018) Study on springback from thermalmechanical boundary condition imposed to V-bending and L-bending processes coupled with infrared rays local heating. Int J Mater Forming 11(3): 417-433. 
5. http://rnfchemical.com

6. Yoon JW, Yang DY, Chung K (1999) Elasto-plastic finite element method based on incremental deformation theory and continuum based shell elements for planar anisotropic sheet materials. Computer Methods in Applied Mechanics and Engineering 174(1-2): 23-56.

7. Kim JB, Yang DY (1998) Finite element analysis of the wrinkling initiation and growth in modified yoshida buckling test, Metals and Materials 4(4): 640-647.

8. https://en.wikipedia.org/wiki/Thermoforming

9. Sridhar R, Rajenthirakumar D (2016) Incremental Forming of PolymerNumerical and Experimental Investigation. Polymers and Polymer Composites,24(7): 489-498.

10. Centeno G, Bagudanch I, Morales-Palma D, García-Romeu ML, Gonzalez-Perez-Somarriba B, et al. (2017) Recent Approaches for the Manufacturing of Polymeric Cranial Prostheses by Incremental Sheet Forming. Procedia Engineering, 183: 180-187.
11. Hwang JS (2016) Investigation into design and manufacture of reinforced lightweight composite sandwich plates based on polymer pyramidal kagome core. Korea Advanced Institute of Science and Technology. Korea.

12. Gutscher G, Wu HC, Ngaile G, Altan T (2004) Determination of flow stress for sheet metal forming using the viscous pressure bulge (VPB) test. J Mater Proc Technol 146(1): 1-7.

13. Liu J, Ahmetoglu M, Altan T (2000) Evaluation of sheet metal formability, viscous pressure forming (VPF) dome test. J Mater Proc Technol 98(1): 1-6.

14. Huang CK, Lou WM, Tsai CJ, Wu TC, Lin HY (2007) Mechanical properties of polymer thin film measured by the bulge test. Thin Solid Films, 515(18): 7222-7226.

15. Bragança IMF, Silva CMA, Alves LM, Martins PAF (2017) Lightweight joining of polymer and polymer-metal sheets by sheet-bulk forming Journal of Cleaner Production, 145(1): 98-104

\section{Your next submission with Juniper Publishers will reach you the below assets}

- Quality Editorial service

- Swift Peer Review

- Reprints availability

- E-prints Service

- Manuscript Podcast for convenient understanding

- Global attainment for your research

- Manuscript accessibility in different formats

( Pdf, E-pub, Full Text, Audio)

- Unceasing customer service

Track the below URL for one-step submission https://juniperpublishers.com/online-submission.php 